\title{
Charmonium production in heavy ion collisions and suggestion of new experiments on fixed target
}

\author{
N.S. Topilskaya* and A.B. Kurepin \\ Institute for Nuclear Research, RAS \\ E-mail: topilska@inr.ru, kurepineinr.ru
}

The experimental data on quarkonium states production measured at the CERN SPS and in $\mathrm{p}-\mathrm{p}$, $\mathrm{p}-\mathrm{Pb}$ and $\mathrm{Pb}-\mathrm{Pb}$ collisions at $\mathrm{LHC}$ and comparison with the data obtained at the Brookhaven National Laboratory Relativistic Heavy Ion Collider RHIC are presented. The suppression of J/ $\psi$ production was suggested as a possible signal of quark gluon plasma formation. The anomalous suppression of $\mathrm{J} / \psi$ production at the CERN SPS was discovered in central $\mathrm{Pb}-\mathrm{Pb}$ collisions by NA50 collaboration at $158 \mathrm{GeV}$. But the effects of $\mathrm{J} / \psi$ suppression on cold nuclear matter and feed-down production from higher charmonium states are important in production of $\mathrm{J} / \psi$. The PHENIX experiment at RHIC at $\sqrt{s}=200 \mathrm{GeV}$ shows that the $\mathrm{J} / \psi$ suppression at these energies is of the same order as the suppression at SPS energies for $\mathrm{Pb}-\mathrm{Pb}$. The models that include regeneration of $\mathrm{J} / \psi$ agree with the experimental results better. The study of charmonium production at LHC in $\mathrm{p}-\mathrm{p}, \mathrm{p}-\mathrm{A}$ and $\mathrm{Pb}-\mathrm{Pb}$ collisions shows the importance of regeneration process. The contribution of B-decay should be taken into account at LHC energy. The high statistic measurements at LHC could investigate the properties of matter at high energy density and temperature. Also the energy intervals between SPS, RHIC and LHC are very important for study of the mechanism of quarkonium production and suppression, in order to investigate medium effects and conditions for Quark Gluon Plasma formation. If the proton and ion beams will be used at LHC with fixed targets, the energy interval between SPS and RICH in p-A and A-A collisions could be investigated. For $7 \mathrm{TeV}$ proton beam we will get $\sqrt{s}=114.6 \mathrm{GeV}$, for Pb beam at $2.75 \mathrm{TeV} \sqrt{s}=$ $71.8 \mathrm{GeV}$. This is unique possibility to clarify the mechanism of charmonium production and to separate two possibilities, first: hard production and suppression in QGP and/or hadronic dissociation or second: hard production and secondary statistical production with $c \bar{c}$ recombination, since the probability of $c \bar{c}$ recombination decreases with decreasing the energy of collision.

XXII International Baldin Seminar on High Energy Physics Problems

15-20 September, 2014

JINR, Dubna, Russia

*Speaker. 


\section{Charmonium production at the CERN SPS}

In ultra-relativistic $\mathrm{Pb}-\mathrm{Pb}$ collisions, quarkonium states production is important probe to study the properties of the deconfined matter, the quark-gluon plasma (QGP). The existing of such a state is predicted by QCD at high temperature and energy density. Since quarkonia are produced at the early stage of the collision, they are expected to interact with the QGP throughout its evolution. The suppression of quarkonium production by colour screening in a dense nuclear matter with different dissociation probabilities for the various mass states, depending on their binding energy was suggested as one of the possible signature of quark-gluon plasma formation in relativistic heavy ions collision [1]. Indeed, at the CERN SPS the NA50 experiment measured the charmonium production in $\mathrm{Pb}-\mathrm{Pb}$ collisions at $158 \mathrm{GeV}$ per nucleon [2] and in p-A collisions at 400 and $450 \mathrm{GeV}$ [3] and observed the normal nucleus suppression of $\mathrm{J} / \psi$ in proton-nucleus reactions and "anomalous" suppression in central lead-lead collisions. The NA60 experiment at SPS measured J/ $\psi$ production in p-A collisions at 400 and $158 \mathrm{GeV}$ and in In-In collisions at $158 \mathrm{GeV}$ per nucleon. It was shown that there are rather strong energy dependence of the absorption cross section and so it is very important to measure charmonium production cross section in $\mathrm{p}-\mathrm{p}, \mathrm{p}-\mathrm{A}$ and $\mathrm{A}-\mathrm{A}$ collisions at the same kinematical domain [4]. The suppression of $\mathrm{J} / \psi$ production by cold nuclear matter $(\mathrm{CNM})$ include not only charmonium absorption at the final state, but also initial state effects. For the first, it is the shadowing effect, i.e. the modification of the parton distribution functions in nuclear matter in comparison with these functions in nucleon. Also, it is necessary to take into account the energy loss of the partons in nuclear matter. So, other nuclear matter effects besides colour screening, as the $\mathrm{J} / \psi$ suppression on cold nuclear matter and feed-down production from higher charmonium states are important in production of J/ $\psi$ at SPS energies. For In-In collisions at $158 \mathrm{GeV}$ per nucleon the additional suppression in central collisions becomes rather small, while for central lead-lead collisions the value of "anomalous" suppression remains near 20 - $30 \%$.

\section{Charmonium production at the Relativistic Heavy Ion Collider RHIC}

PHENIX experiment at the collider RHIC measured $\mathrm{J} / \psi$ production in $\mathrm{p}-\mathrm{p}, \mathrm{d}-\mathrm{Au}, \mathrm{Au}-\mathrm{Au}$ and $\mathrm{Cu}-\mathrm{Cu}$ collisions at $\sqrt{s}=200 \mathrm{GeV}$ energy for the N-N centre of mass system [5,6]. The suppression of the $\mathrm{J} / \psi$ production was calculated as a ratio of $\mathrm{J} / \psi$ production in A-A collisions, normalized to the number of binary collisions, $N_{\text {coll }}$, to the $\mathrm{J} / \psi$ production in p-p collisions. This $R_{A A}$ ratio (the nuclear modification factor), where $R_{A A}=d N_{A A} / d y /\left(d N_{p p} / d y *\left\langle N_{\text {coll }}>\right.\right.$ ), was used for comparison of the experimental data versus centrality of collisions, multiplicity, number of participant, $N_{\text {part }}$, energy density, transverse momentum and so on. The suppression of the $\mathrm{J} / \psi$ production in Au-Au collisions in forward rapidity range $1.2<|y|<2.2$ was found to be stronger than suppression in rapidity range $|y|<0.35$. For the most central events the suppression of the $\mathrm{J} / \psi$ production achieves the value near $80 \%$. Recently at PHENIX also was measured the $\mathrm{J} / \psi$ production in U-U collisions at $\sqrt{s}=198 \mathrm{GeV}$. The suppression of charmonium production is approximately the same for all $\mathrm{Au}-\mathrm{Au}, \mathrm{Cu}-\mathrm{Cu}$ and $\mathrm{U}-\mathrm{U}$ colliding systems. Measuring the charmonium production in $\mathrm{Au}-\mathrm{Au}$ collisions at lower energies 62.4 and $39 \mathrm{GeV}$ also reveals approximately the same $\mathrm{J} / \psi$ suppression as at $200 \mathrm{GeV}$, but has large statistical errors due to low luminosity and large systematical errors because of absence of p-p collisions at the same energies [7, 8]. There are several theoretical models which could describe experimental data with and without taking into account the regen- 
eration of $\mathrm{J} / \psi$, but there predictions for the $\mathrm{J} / \psi$ production at LHC energy are different - from large suppression in the model [9] to the enhancement in [10]. STAR experiment at RHIC also measured the $\mathrm{J} / \psi$ production in $\mathrm{Au}$-Au collisions at large transverse momentum $p_{\mathrm{T}}>5 \mathrm{GeV} / \mathrm{c}$ for mid-rapidity range. The suppression for the $\mathrm{J} / \psi$ production is smaller than measured at PHENIX and consists near $50 \%$ for the most central collisions [11]. For extractions the CNM effect the PHENIX data for $\mathrm{Au}-\mathrm{Au}$ and d-Au collisions were analyzed simultaneously. The nuclear modification factor for cold nuclear matter $R_{A A}(C N M)$ in Au-Au collisions at measured rapidity ranges was obtained. The ratio $R_{A A} / R_{A A}(C N M)$ shows the "anomalous" suppression of the $\mathrm{J} / \psi$ production in the hot and dense nuclear matter produced in relativistic heavy ions collisions. At $\sqrt{s}=$ $200 \mathrm{GeV}$ the $R_{A A} / R_{A A}(C N M)$ ratio becomes approximately equal for different ranges of rapidity and consists near $50 \%$ for the most central events. Comparison of NA50, NA60 and PHENIX data [12] versus multiplicity shows that at RHIC energy "anomalous" suppression of J/ $\psi$ for all colliding nuclei is the same as at SPS energy. But the dependence of "anomalous" J/ $\psi$ suppression on $N_{\text {part }}$ shows that suppression in Au-Au collisions at RHIC is stronger that suppression in $\mathrm{Pb}-\mathrm{Pb}$ collisions at SPS (fig1).
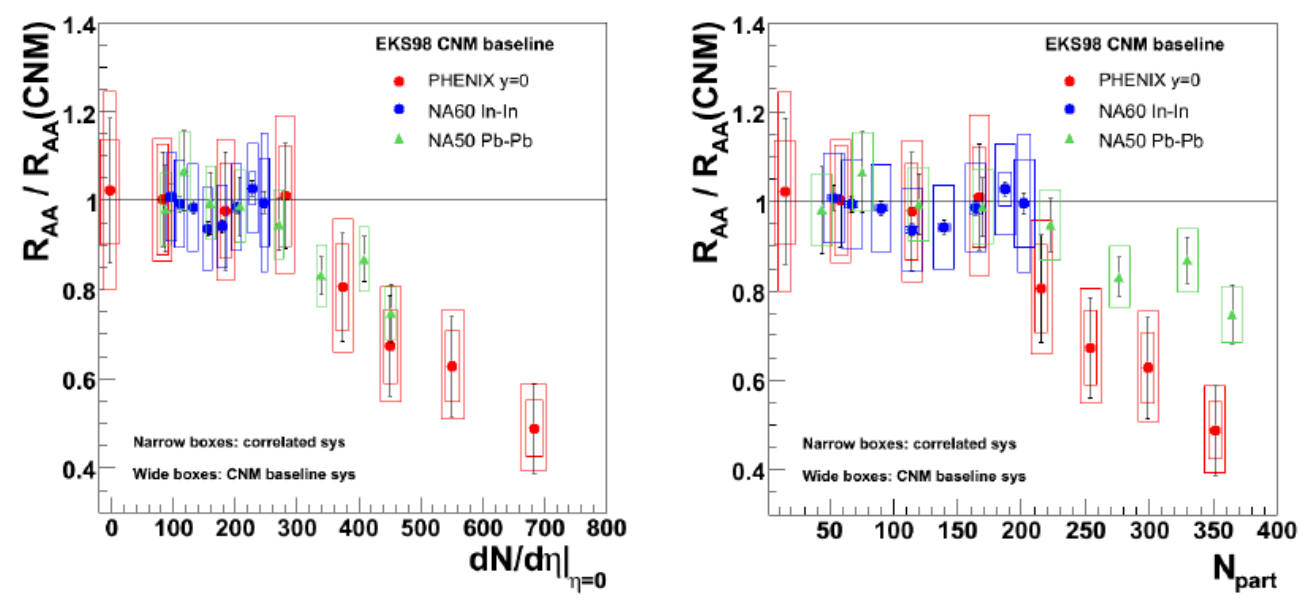

Figure 1: The comparison of NA50, NA60 and PHENIX $R_{A A}$ results versus multiplicity (left panel) and versus $N_{\text {part }}$ (right panel).

So it is important to choose the correct parameter for comparison of the data. It was suggested that in p-p, p-A and d-Au collisions at SPS and RHIC energies the hot and dense nuclear matter did not exist and suppression of charmonium production are produced only by cold nuclear matter. But it may be not the case at the higher energies.

\section{Quarkonium production at the CERN Large Hadron Collider LHC}

At LHC energies five quarkonium states from two families are under study, charmonia: $\mathrm{J} / \psi$ and $\psi(2 S)$ and bottomonia: $\Upsilon(1 S), \Upsilon(2 S)$ and $\Upsilon(3 S)$. In the colour-screening model quarkonium states are suppressed in the QGP with different dissociation probabilities for the various mass states, depending on their binding energy. The CMS Collaboration at the LHC has reported on the observation of the sequential suppression of bottomonium states in $\mathrm{Pb}-\mathrm{Pb}$ collisions at $\sqrt{s_{N N}}=2.76 \mathrm{TeV}$ 
$[13,14]$. However, other nuclear matter effects besides colour screening, as well as cold nuclear matter (CNM) effects, regeneration and feed down from higher states and B-meson decay for $\mathrm{J} / \psi$ production do complicate this simple picture. Recent measurements by the ALICE Collaboration are compatible with a regeneration mechanism playing an important role in the production of $\mathrm{J} / \psi$ in $\mathrm{Pb}-\mathrm{Pb}$ collisions at the LHC $[15,16,17]$. Additional $\mathrm{J} / \psi$ are expected to be produced from deconfined charm quarks by kinetic recombination in the QGP [9] or by statistical hadronization at the phase boundary [10]. This additional, hot nuclear matter effect, competes with the suppression by colour screening. Due to the lower production cross section of $b \bar{b}$ pairs compared to $c \bar{c}$ pairs, the regeneration of $\Upsilon(1 \mathrm{~S})$ is expected to be smaller than that of $\mathrm{J} / \psi$ [18]. On the other hand, effects related to the presence of CNM can also modify the production of quarkonia in nucleus-nucleus collisions. Cold nuclear matter effects can be separated into initial and final-state effects. Initialstate effects include shadowing and parton energy loss. The nuclear Parton Distribution Function (nPDF) differ from those in free nucleons (PDF). Since the gluon fusion mechanism dominates in heavy-quarks production, quarkonium production is particularly sensitive to the gluon $\mathrm{nPDF}$, which is presently not well known. Bjorken parameter $x_{B}$ is defined as the fraction of the hadron momentum carried by the parton. If the quarkonium production is dominated by low $x_{B}$ gluons, then the Colour Glass Condensate (CGC) model can be used to describe the nucleus as a coherent gluonic system that saturates at very large density [19]. Finally, partons can lose energy before creating the heavy-quark pair, therefore modifying the kinematic distributions of quarkonia. Final-state effects affect the heavy-quark pair during formation quarkonium state or after the state has been formed. The heavy-quark pair can interact with the nuclear matter and eventually break up, that is normal nuclear absorption. The final-state resonance can also interact with surrounding comovers and lose energy or even break up. The break up cross section depends on the nature of state and expected to be small at high energy. The LHC collider at CERN now gives the possibility to measure quarkonium production at the energy range more than ten times higher than at RHIC. In four experiments ALICE [20], ATLAS [21], CMS [22] and LHCb [23] at LHC, the quarkonium production was measured in different rapidity and transverse momentum ranges. The $\mathrm{J} / \psi$ production was measured in p-p collisions at 2.76, 7 and $8 \mathrm{TeV}$ and in $\mathrm{Pb}-\mathrm{Pb}$ collisions at 2.76 TeV. The quarkonium production in $\mathrm{p}-\mathrm{Pb}$ collisions at $5.02 \mathrm{TeV}$ was measured in 2013 year for detailed investigation of the cold nuclear matter effects and investigation of production mechanism.

\subsection{Charmonium production at the LHC}

Measurement of the $\mathrm{J} / \psi$ production in p-p collision at the same energy as in $\mathrm{Pb}-\mathrm{Pb}$ collision, gives the reference for extracting the nuclear modification factor $R_{A A}$. The mechanism of $\mathrm{J} / \psi$ production is investigated and the contribution of $\mathrm{B}$ - decay to $\mathrm{J} / \psi$ production is measured. There is a good agreement for p-p collision between the data obtained in ALICE, LHCb, CMS and ATLAS experiments in the same kinematical domains [24]. The $J / \psi$ production cross section depends on rapidity. For forward rapidity the cross section is smaller. By increasing the energy the mean transverse momentum and production cross section of $\mathrm{J} / \psi$ become larger. The contribution of Bdecay to $\mathrm{J} / \psi$ production cross section depends on rapidity and increases for larger $\mathrm{J} / \psi$ transverse momentum. This contribution is approximately $10 \%$ for $p_{\mathrm{T}}$ near $1.5 \mathrm{GeV} / \mathrm{c}$ [25].

Charmonium production in $\mathrm{Pb}-\mathrm{Pb}$ collisions at $2.76 \mathrm{TeV}$ was measured at LHC in ALICE, CMS and ATLAS experiments. In ALICE experiment the transverse momentum of inclusive $\mathrm{J} / \psi$ 
mesons was measured from values near zero up to $8 \mathrm{GeV} / \mathrm{c}$ in rapidity range $|y|<0.9$ (for $\mathrm{J} / \psi$ decay into two electrons) and $2.5<y<4$ (for muon channel). In ATLAS and CMS experiments charmonium production was measured in the rapidity range $|y|<2.4$, but the range of transverse momentum values depended on rapidity. In ATLAS experiment only $\mathrm{J} / \psi$ mesons with large transverse momentum $p_{\mathrm{T}}>6.5 \mathrm{GeV} / \mathrm{c}$ were measured. The comparison of PHENIX and ALICE $R_{A A}$ results is shown in fig2 $[24,6]$.
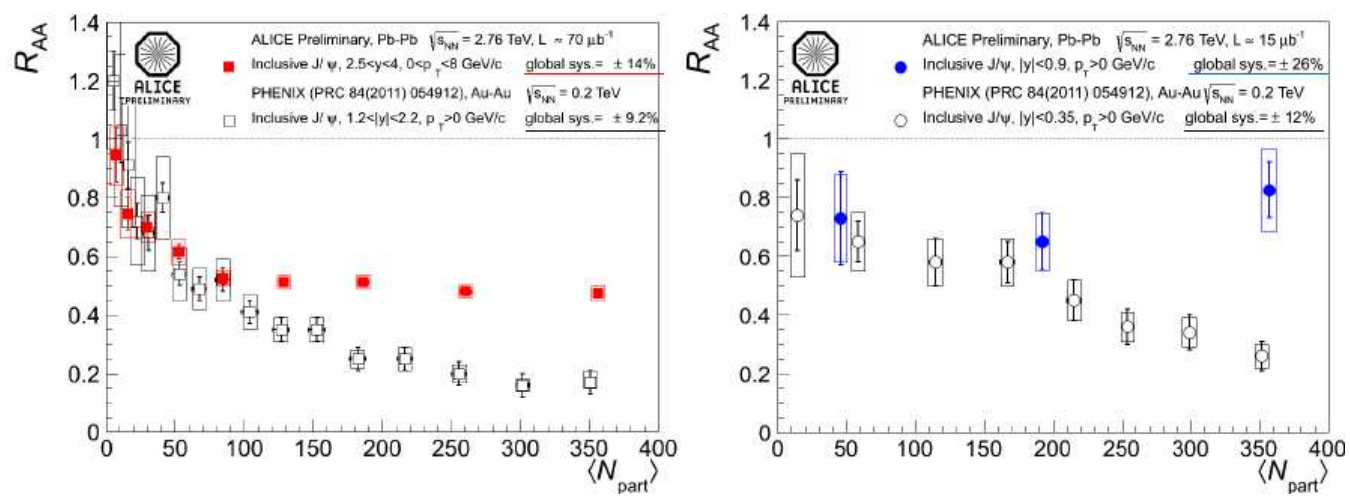

Figure 2: The comparison of PHENIX and ALICE $R_{A A}$ results. The two panels show the data at forward rapidity (left) and mid-rapidity (right).

The ALICE measurement of the inclusive $\mathrm{J} / \psi$ production in $\mathrm{Pb}-\mathrm{Pb}$ collisions at $\sqrt{s_{N N}}=$ $2.76 \mathrm{TeV}$ has shown less suppression compared to PHENIX results in Au-Au collisions at $\sqrt{s_{N N}}=$ $0.2 \mathrm{TeV}$ [17]. At $\sqrt{s_{N N}}=2.76 \mathrm{TeV}$, the charm quark density produced in the collisions increases with respect to SPS and RHIC energies. This may enhance the probability to create $J / \psi$ from recombination of charm quarks. If the $\mathrm{J} / \psi$ mesons are fully suppressed in QGP, their creation will take place at chemical freeze-out [9]. If $\mathrm{J} / \psi$ mesons survive in the QGP, production will take place continuously during the lifetime of QGP [10]. The probability of $c \bar{c}$ recombination increases with increasing the energy of collision and at LHC energies may become dominant. This contribution leads to an increase of the $R_{A A}$ at the LHC with respect to RHIC. In particular, this mechanism predicts an increase of the $R_{A A}$ from forward- to mid-rapidity, where the density of charm quarks is higher. Moreover, in order to recombine, two charm quarks need to be close enough in phase space, so the effect will be larger at low transverse momentum of $\mathrm{J} / \psi$. So the transverse momentum and rapidity dependence distributions of the $\mathrm{J} / \psi R_{A A}$ are important for deeper understanding the mechanism of charmonium production.

Inclusive $\mathrm{J} / \psi$ production has been studied at the LHC in $\mathrm{p}-\mathrm{Pb}$ collisions at energy $\sqrt{s_{N N}}=$ $5.02 \mathrm{TeV}$. At ALICE the measurement is performed in the center of mass rapidity domains $2.03<$ $y<3.53$ and $-4.46<y<-2.96$ in muon channel down to zero transverse momentum [28]. Since p-p collisions data at $\sqrt{s}=5.02 \mathrm{TeV}$ for determination of $R_{p P b}$ are not available, the reference $\sigma_{p p}(J \psi)$ has been obtained by an interpolation procedure, based on forward rapidity p-p collisions results at $\sqrt{s}=2.76$ and $7 \mathrm{TeV}$ from ALICE. While at forward rapidity, corresponding to the proton direction, a suppression of the $\mathrm{J} / \psi$ yield with respect to binary-scaled p-p collisions is observed, in the backward region no suppression is presented. The $\mathrm{J} / \psi$ production in $\mathrm{p}$-A collisions was also measured in electron channel in the center of mass rapidity domains $-1.37<y<0.43$ [28]. The 
experimental results in comparison with $\mathrm{LHCb}$ results are shown in fig3 (left panel).
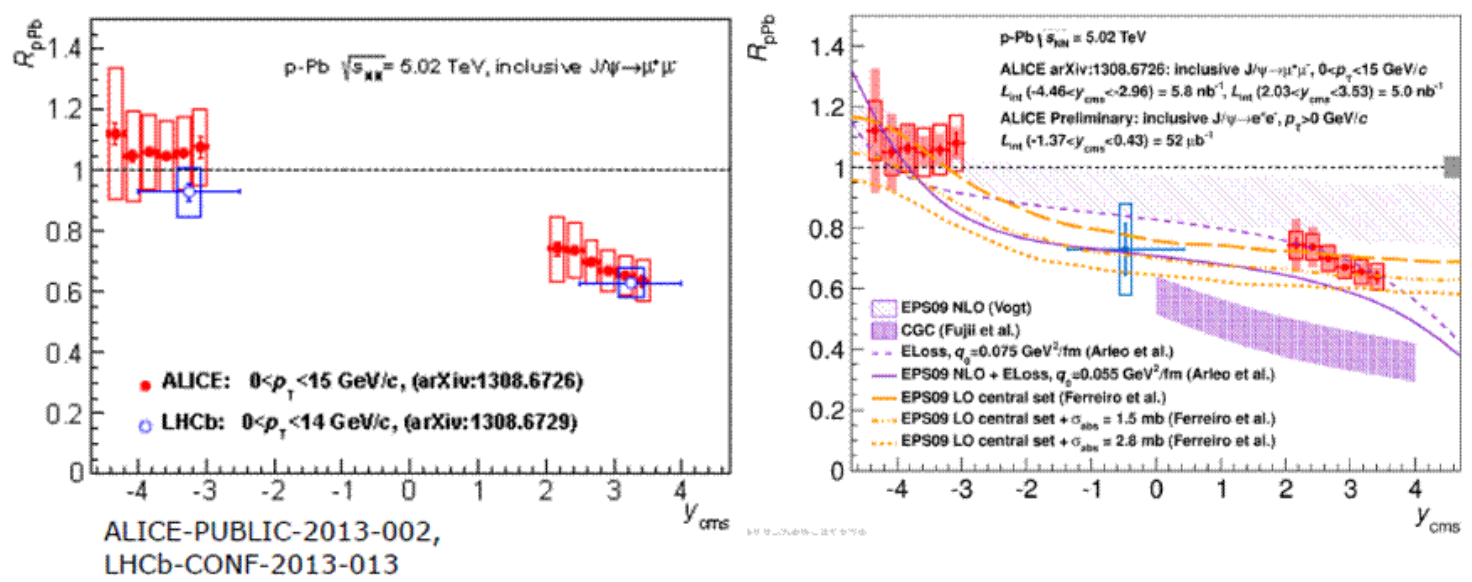

Figure 3: The comparison of ALICE and LHCb $R_{p P b}$ results for $\mathrm{J} / \psi$ (left panel) and the comparison of ALICE $R_{p P b}$ results with theoretical predictions (right panel).

The ALICE and LHCb results are in good agreement. Theoretical predictions based on nuclear shadowing as well as on models including, in addition, a contribution from partonic energy loss, are in fair agreement with the experimental results. The Colour Glass Condensate (CGC) model could not reproduce data (fig3, right panel).

The measuring of $\psi(2 \mathrm{~S})$ production also was performed in $\mathrm{p}-\mathrm{Pb}$ collisions at $\sqrt{s}=5.02 \mathrm{TeV}$ in ALICE. The nuclear modification factor for inclusive $\psi(2 S)$ is evaluated and compared to the measurement of the same quality for $\mathrm{J} / \psi$ and to theoretical models including parton shadowing and coherent energy loss mechanism [26]. The results show a significantly larger suppression of the $\psi(2 \mathrm{~S})$ compared to that measured for $\mathrm{J} / \psi$ and to models (fig4).
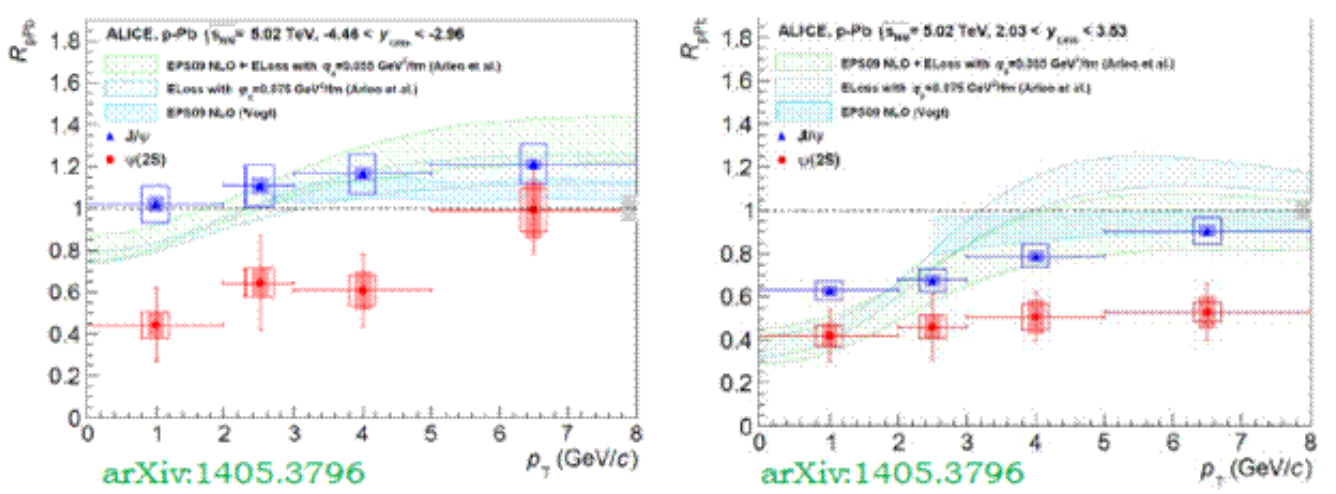

Figure 4: The comparison of ALICE $R_{p A}$ results for $\psi(2 \mathrm{~S})$ with theoretical predictions

These observations represent a clear indication for significant final state effects on $\psi(2 \mathrm{~S})$ production. 


\subsection{Bottomonium production at the $\mathrm{LHC}$}

The inclusive production cross sections of $\Upsilon(1 S)$ and $\Upsilon(2 S)$ as a function of $p_{\mathrm{T}}$ and rapidity have been measured using ALICE detector at forward rapidity in p-p collisions at a centre of mass energy $\sqrt{s}=7 \mathrm{TeV}$ [29]. The results are in good agreement with measurements from LHCb experiment $[23,30]$ over similar $p_{\mathrm{T}}$ and rapidity ranges. They are complemented to CMS measurements at mid-rapidity [31,32]. They are also in good agreement with NRQCD calculations. The CMS Collaboration at the LHC has reported on the observation of the sequential suppression of bottomonium states in $\mathrm{Pb}-\mathrm{Pb}$ collisions at $\sqrt{s_{N N}}=2.76 \mathrm{TeV}[13,14]$. The $\Upsilon(1 \mathrm{~S})$ yield is suppressed by approximately a factor of two with respect to the expectation from p-p collisions obtained by scaling with the number of binary nucleon-nucleon collisions. The $\Upsilon(2 S)$ and the $\Upsilon(3 S)$ are almost completely suppressed.

The ALICE measurement of inclusive $\Upsilon(1 \mathrm{~S})$ production in $\mathrm{Pb}-\mathrm{Pb}$ collisions was carried out at forward rapidity and down to zero transverse momentum using muon decay channel [33]. A strong suppression is observed with respect to $p$-p collisions scaled by the number of independent nucleon-nucleon collisions (fig5).
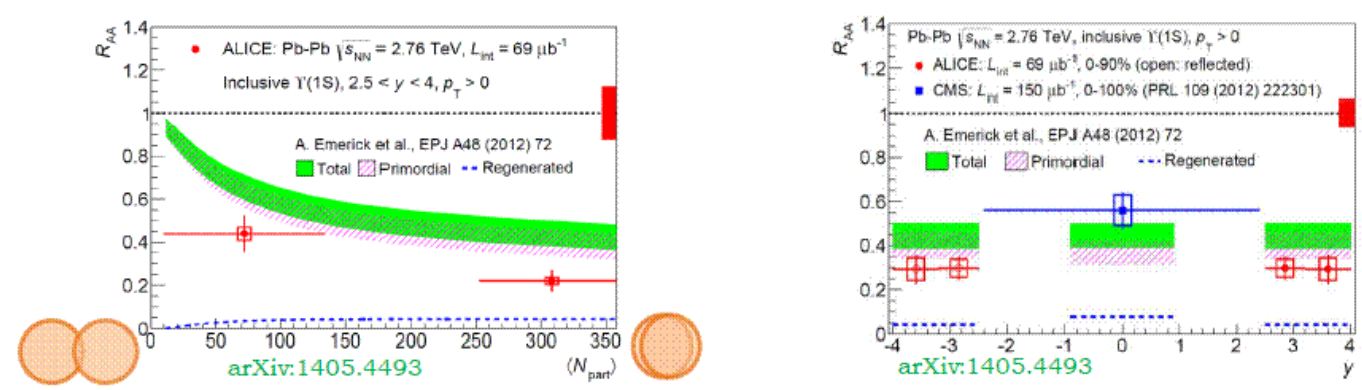

Figure 5: The comparison with theoretical predictions ALICE $R_{P b P b}$ results for $\Upsilon(1 \mathrm{~S})$ versus $N_{\text {part }}$ (left panel) and ALICE and CMS results versus rapidity (fight panel).

The observed suppression increases with centrality of the collisions and seems be more that in mid-rapidity measurements. The theoretical models underestimate the measured suppression and fail to reproduce its rapidity dependence.

The ALICE results are compared with the calculation from a transport model [18]. The transport model employs a kinetic rate equation approach in an evolving QGP and includes both suppression and regeneration effects. CNM effects were calculated by varying effective absorption cross section. The transport model clearly underestimates the observed suppression, but the shape of the centrality dependence is fairly reproduced. The ALICE results are compared with those of CMS $(|y|<2.4$. The observed suppression is stronger at forward than at mid-rapidity (fig.5). The model [18] predicts a nearly constant $R_{A A}$ which is in disagreement with ALICE and CMS data.

The production of inclusive $\Upsilon(1 \mathrm{~S})$ and $\Upsilon(2 \mathrm{~S})$ was measured also in $\mathrm{p}$ - $\mathrm{Pb}$ collisions at $\sqrt{s}$ $=5.02 \mathrm{TeV}$ in ALICE. A suppression of the inclusive $\Upsilon(1 \mathrm{~S})$ yield in $\mathrm{p}-\mathrm{Pb}$ collisions with respect to the yield from p-p collisions scaled by the number of binary nucleon-nucleon collisions is observed at forward rapidity but not at backward rapidity (fig6).

The results are compared to theoretical model calculations including nuclear shadowing or/and 


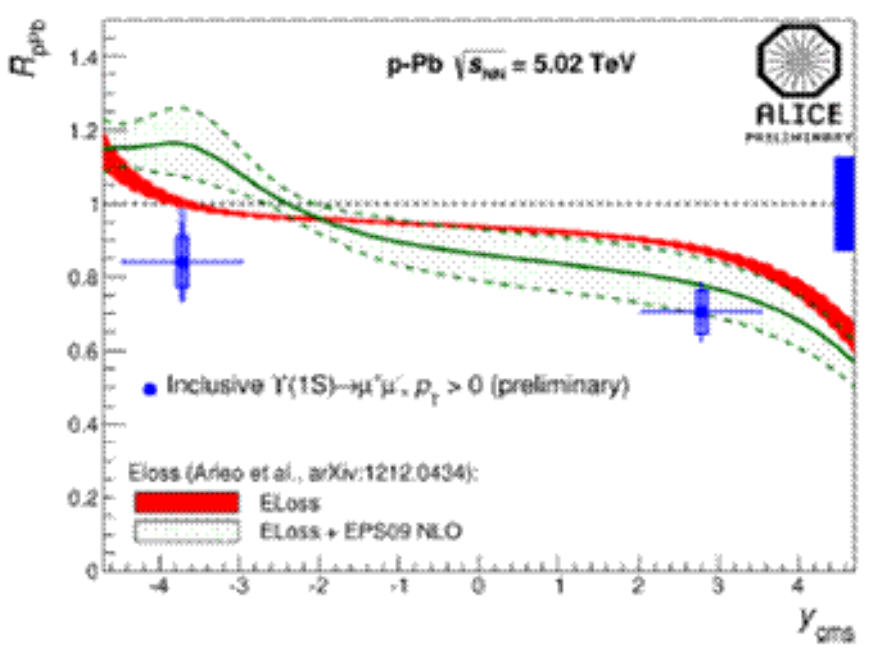

Figure 6: The comparison of ALICE $R_{p P b}$ results for $\Upsilon(1 \mathrm{~S})$ versus rapidity with theoretical predictions

partonic energy loss effects. Only models with energy loss plus shadowing could describe the data at forward rapidity but these models underestimate the suppression at backward rapidity.

Quarkonium production at LHC is a useful probe for the investigation of QGP formation and for testing pQCD models in p-p scattering. Indeed, $\mathrm{J} / \psi, \psi(2 \mathrm{~S})$ and $\Upsilon(1 \mathrm{~S})$ differential cross sections in p-p collisions could be described by NLO NRQCD models. For p-Pb collisions theoretical models including nuclear suppression, parton shadowing and coherent energy loss mechanisms could reproduce $\mathrm{J} / \psi$ production, but fail to describe additional suppression of $\psi(2 \mathrm{~S})$ and underestimate the observed $\Upsilon(1 \mathrm{~S})$ suppression at forward rapidity. In $\mathrm{Pb}-\mathrm{Pb}$ collisions the evidence for strong $\mathrm{J} / \psi$ suppression at large transverse momentum and additional $\mathrm{J} / \psi$ production from regeneration at low $p_{\mathrm{T}}$ was obtained.

\section{Quarkonium production at fixed target at LHC beams}

Progress in our understanding of the properties of matter requires systematic and high statistic measurements also in low energy region. The energy intervals between SPS, RHIC and LHC are very important to study the mechanism of quarkonium production and suppression, in order to investigate medium effects and conditions of quark-gluon plasma formation. Unfortunately, beam energy scan at RHIC produced data with low statistic and large systematic errors because the luminosity at RHIC decreases with energy reduction. It is important to study the mechanism of quarkonium production and suppression at low energies up to $35 \mathrm{GeV} /$ per nucleon at the FAIR in CBM and at NICA collider in Dubna with high statistic. At CERN SPS the fixed target experiment CHIC for charmonium study at energy up to $20 \mathrm{GeV}$ is under preparation. At RHIC there are suggestions for experiments at fixed target with PHENIX and STAR installations.

If the proton and ion beams would be used at LHC with fixed targets, the energy interval between SPS and RHIC in p-A and A-A collisions could be covered. For $7 \mathrm{TeV}$ proton beam, the energy in N-N c.m. is $\sqrt{s}=114.6 \mathrm{GeV}$, for Pb beam at $2.75 \mathrm{TeV}$ it is $\sqrt{s}=71.8 \mathrm{GeV}$. Using LHC beams with reduced energy it would be possible to scan energy range between SPS and RHIC. 
This is a unique possibility to clarify the mechanism of charmonium, $\mathrm{J} / \psi$ and $\psi(2 \mathrm{~S})$ production, to separate two possibilities, first: hard production and suppression in QGP and/or hadronic dissociation or second: hard production and secondary statistical production with recombination, since the probability of recombination decreases with decreasing energy of collision in thermal model [34]. In order to study the feasibility of using the fixed target at LHC for charmonium production, the geometrical acceptances for $\mathrm{J} / \psi$ production on fixed target by means of AliRoot - FAST simulations were obtained. As described in [35] for quarkonium production the phenomenological Colour Evaporation Model (CEM) was used. The rapidity and transverse momentum distributions for "prompt" $\mathrm{J} / \psi$ production are obtained respectively as a parameterization of the CEM predictions and by extrapolating to LHC energy the $\mathrm{J} / \psi$ transverse momentum $p_{\mathrm{T}}$ distribution, measured at mid-rapidity by the CDF experiment at c.m. energy near $1.8 \mathrm{TeV}$. The geometrical acceptances of J/ $\psi$ production for PHENIX at RHIC and fixed target experiments NA50 at SPS and HERA-B are calculated for comparison.

The calculated geometrical acceptances for fixed target measurement are of the same order and even larger than geometrical acceptances for colliding nuclei in ALICE. The counting rates are calculated and it is shown that the $\mathrm{J} / \psi$ production on fixed targets at LHC could be measured with high statistics collected in several days of data taking [35].

\subsection{Luminosity, cross sections and counting rates for $\mathrm{p}-\mathrm{p}, \mathrm{p}-\mathrm{A}$ and $\mathrm{Pb}-\mathrm{Pb}$ collisions}

As it was already used for the experiment on collider with a fixed target at HERA-B [36], the target in the form of thin ribbon could be placed around the main orbit of LHC. The life time of the beam is determined by the beam-beam and beam-gas interactions. Therefore after some time the particles will leave the main orbit and interact with the target ribbon. So for fixed target measurements only halo of the beam will be used. Therefore no deterioration of the main beam will be introduced. The experiments at different interaction points will not feel any presence of the fixed target at the IP of ALICE. The luminosity estimate is shown in the Table 1.

From experimental ALICE 2011 year p-p run we got 1.2. $10^{11}$ protons per bunch, 1380 bunches and life time 14.5 hours. From these parameters we get particle loss of $1.1 \cdot 10^{13}$ protons during one hour $\left(3.1 \cdot 10^{9}\right.$ protons/s) and mean luminosity about $3 \cdot 10^{30} \mathrm{~cm}^{-2} \mathrm{~s}^{-1}$ for 500 micron lead ribbon. For $\mathrm{Pb}$ beam we got $1 . \cdot 10^{8}$ ions per bunch, 358 bunches and life time 6.5 hours. We get particle loss of $5.1 \cdot 10^{9}$ ions during one hour $\left(1.4 \cdot 10^{6}\right.$ ions/s) and mean luminosity about $1.7 \cdot 10^{27} \mathrm{~cm}^{-2} \mathrm{~s}^{-1}$ for 500 micron lead ribbon.

Since the target ribbon should not interfere during the beam formation and acceleration process it should be lifted in the working position after the tuning of the beam. The value of the nucleonnucleon charmonium total production cross section shown in the Table 1 for $14 \mathrm{TeV}$ was calculated by CEM model with MRST HO PDF. The cross sections for lower energies were obtained by interpolation of the measured at RHIC proton-proton collision at $\sqrt{s}=200 \mathrm{GeV}$, from NA51 p-p and p-d experiment at $450 \mathrm{GeV}$ per nucleon $(\sqrt{s}=29.1 \mathrm{GeV})$ and extracted from the data of NA50 experiment at $\sqrt{s}=27.4 \mathrm{GeV}$ for proton-lead collisions.

With the counting rate values presented in Table 1 for $\mathrm{p}-\mathrm{A}$ and A-A collisions with fixed target high statistical results could be obtained for possible measurements of $\mathrm{J} / \psi$ production. For A-A collisions luminosity is smaller but the production cross section is larger. Therefore the counting 
Table 1: Luminosity, cross sections $\left(x_{F}>0\right)$ and counting rates.

\begin{tabular}{|c|c|c|c|c|c|c|c|}
\hline System & $\sqrt{s}, \mathrm{TeV}$ & $\sigma_{n n}, \mu \mathrm{b}$ & $\begin{array}{c}\sigma_{p A}, \mu \mathrm{b} \\
\left(A^{0.92} \sigma_{n n}\right)\end{array}$ & $\mathrm{I}, \%$ & $\mathrm{IB} \sigma_{p A}, \mu \mathrm{b}$ & $\mathrm{L}, c^{-2} s^{-1}$ & Rate, $h^{-1}$ \\
\hline $\mathrm{pp}$ & 14 & 54.1 & 54.1 & 4.7 & 0.150 & $3 \cdot 10^{30}$ & 1620 \\
$\mathrm{pp}_{\text {RHIC }}$ & 0.200 & 2.7 & 2.7 & 3.59 & 0.0057 & $1 \cdot 10^{31}$ & 205 \\
$\mathrm{pPb}_{\text {NA50 }}$ & 0.0274 & 0.19 & 25.7 & 14.0 & 0.212 & $7 \cdot 10^{29}$ & 535 \\
$\mathrm{pPb}_{\text {fixed }}$ & 0.1146 & 0.65 & 80.2 & 5.98 & 0.310 & $3 \cdot 10^{30}\left(^{\star}\right)$ & 3360 \\
$\mathrm{pPb}_{\text {fixed }}$ & 0.0718 & 0.55 & 74.6 & 7.97 & 0.349 & $3 \cdot 10^{30}$ & 3780 \\
$\mathrm{PbPb}_{\text {fixed }}$ & 0.0718 & 0.55 & 11970 & 7.97 & 42.9 & $1.7 \cdot 10^{27}\left({ }^{\star \star}\right)$ & 292 \\
\hline
\end{tabular}

$\left(^{\star}\right)-\mathrm{pPb}_{\text {fixed }}, 500 \mu$ wire, $3.1 \cdot 10^{9}$ protons/s. $\left(^{\star \star}\right)-\mathrm{PbPb}_{\text {fixed }}, 500 \mu$ wire, $1.4 \cdot 10^{6}$ ions/s.

rate is of the same order. It is also clear that the measurement of $\psi(2 \mathrm{~S})$ production is feasible with better statistical accuracy than at RHIC collider.

Later French group of physicists also suggests fixed target experiment AFTER at LHC beams using extracted with strong crystalline field part of the beam [37]. The fixed target experiment provides precise quarkonium studies and has advantage of high luminosity and measuring of cold nuclear matter effects what is strongly limited at collider. The use of fixed target at LHC could provide in a short time the data for different targets and maybe for different projectile nuclei with high statistics. Therefore the important information about mechanism of charmonium production and possible QGP formation could be obtained.

\section{References}

[1] T. Matsui and H. Satz, Phys. Lett. B178, 416 (1986).

[2] B. Alessandro et al., NA50 Collaboration, Eur. Phys. J C39, 335 (2005) and references therein.

[3] B. Alessandro et al., NA50 Collaboration, Phys. Lett. B553, 167 (2003).

[4] R. Arnaldi et al., NA60 Collaboration, Nucl. Phys. A830, 345c (2009); Eur. Phys. J C59, 607 (2009).

[5] A. Adare et al., PHENIX Collaboration, Phys. Rev. C84, 054912 (2011); arXiv :1103.6269 v1.

[6] A. Adare et al., PHENIX Collaboration, Phys. Rev. D85, 092004 (2012); arXiv :1105.1966.

[7] B.I. Abelev et al., STAR Collaboration, Phys. Rev. C80, 41902 (2009).

[8] A. Adare et al., PHENIX Collaboration, Phys. Rev. C86, 064901 (2012).

[9] R. L. Thews et al., Eur. Phys. J C43, 97 (2005); R. L. Thews and M.I. Mangano, Phys. Rev. C73, 014904 (2006).

[10] A. Andronic, P. Braun-Munzinger, K. Redlich, and J. Stachel, Phys. Rev. Lett. 659, 149 (2008).

[11] H. Masui and Z. Tang, STAR collaboration, J. Phys. G38, 124002(2011).

[12] H. Brambilla et al., Eur. Phys. J C71, 1534 (2011).

[13] S. Chatrchyan et al., CMS Collaboration, Phys. Rev. Lett. 109, 222301 (2012).

[14] S. Chatrchyan et al., CMS Collaboration, Phys. Rev. Lett. 107, 052302 (2011). 
[15] B. Abelev et al., ALICE collaboration, Phys. Rev. Lett. 109, 072301 (2012).

[16] E. Abbas et al., ALICE collaboration, Phys. Rev. Lett. 111, 162301 (2013).

[17] B. Abelev et al., ALICE collaboration, Phys. Lett. B743, 314 (2014).

[18] A. Emerick, X. Zhao and R. Rapp, Eur. Phys. J A48, 72 (2012).

[19] H. Fujii, K. Watanabe, Nucl. Phys. A915,1 (2013).

[20] K. Aamodt et al., ALICE collaboration, Phys. Lett. B704, 442(2011).

[21] G. Aad et al., ATLAS collaboration, Nucl. Phys. B850, 387(2011); arXiv:1012.5419 v1.

[22] V. Khachatryan et al., CMS Collaboration, Eur. Phys. J C71, 1675 (2011); arXiv:1011.4193.

[23] R. Aaij et al., LHCb collaboration, Eur. Phys. J C71, 1645(2011); arXiv:1103.0423 v2.

[24] P. Pillot, ALICE Collaboration, J. Phys. G38, 124111 (2011).

[25] T. Dahms, CMS Collaboraion, J. Phys. G38, 124105 (2011).

[26] B. Abelev et al., ALICE collaboration, JHEP 12, 073 (2014).

[27] B. Abelev et al., ALICE collaboration, Phys. Lett. B740, 105 (2015).

[28] B. Abelev et al., ALICE collaboration, JHEP 02, 073 (2014).

[29] B. Abelev et al., ALICE collaboration, Eur. Phys. J C73, 2662(2013).

[30] R. Aaij et al., LHCb collaboration, Eur. Phys. J C72, 2100(2012).

[31] V. Khachatryan et al., CMS Collaboration, Phys. Rev. D23, 112004 (2011).

[32] S. Chatrchyan et al., CMS Collaboration, Phys. Lett. B727, 101 (2013).

[33] B. Abelev et al., ALICE collaboration, Phys. Rev. Lett. B738, 361 (2014).

[34] R. Hagedorn, Riv. Nuovo Cimento 6, 1 (1983).

[35] A. B. Kurepin, N. S. Topilskaya and M. B. Golubeva, Phys. Atom. Nucl. 74, 446 (2011) and references therein.

[36] K. Ehret, Nucl. Instr. Meth A446, 190 (2000).

[37] J.P.Lansberg et al., arXiv: 1202.6585. 